Boletín de la Sociedad Geológica Mexicana

VOLUMEN 64, NÚM. 3, 2012, P. 295-304

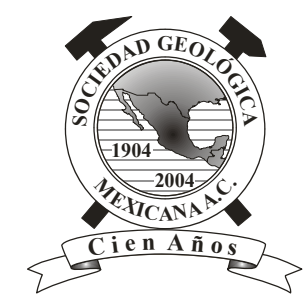

\title{
Caracterización Petrofísica de arenas del Neógeno en un pozo gasífero de la Cuenca de Veracruz
}

\author{
Antonio Quilantan Pedraza ${ }^{1, *}$, Marcos Antonio Reyes Sánchez ${ }^{2}$ \\ ${ }^{1}$ Activo Regional de Exploración, PEMEX Exploración y Producción, Poza Rica, Veracruz. \\ ${ }^{2}$ Activo Integral Veracruz. PEMEX Exploración y Producción, Veracruz, Veracruz. \\ *geoquilantan@hotmail.com
}

\section{Resumen}

Para conocer el potencial productor de hidrocarburos en un yacimiento y la optimización del mismo es necesario la aplicación de diferentes ingenierías y disciplinas, una de las disciplinas importantes es la petrofísica, que realiza la caracterización petrofísica del yacimiento a un nivel estático, la cual determina las propiedades físicas de las rocas y tipos de fluidos presentes en el yacimiento principalmente: porosidad, permeabilidad, tamaño de grano, de poro y saturación de fluidos. Para determinar las propiedades petrofísicas se realiza una evaluación de registros geofísicos, integrando datos de núcleo. En el área de estudio se identificaron 5 horizontes con potencial gasífero a nivel del Neógeno, los cuales comprenden 7 tipos de roca petrofísicos y 13 unidades de flujo. La caracterización petrofísica ayudó a reevaluar las reservas, definir los tipos de roca y unidades de flujo que contribuyen a la producción, así como identificar zonas con potencial de producción en horizontes arenosos correlacionables al área de estudio.

Palabras clave: evaluación de registros, tipos de roca petrofísicos y unidades de flujo.

\begin{abstract}
To know the output potential of hydrocarbons in a reservoir and its optimization, it is necessary to apply different engineering disciplines. One important discipline is petrophysics, which carries out the petrophysical characterization of the reservoir at a static level, and determines the physical properties of the rocks and types of fluids present in the reservoir, mainly porosity, permeability, grain size, and pore and fluid saturation. To determine the petrophysical properties, a geophysical log evaluation is carried out, integrating core data. Five horizons with gas potential were identified at the study area at the Neogene level, which include seven types of petrophysical rock types and thirteen flow units. The petrophysical description helped to re-evaluate the reserves and define the rock types and flow units that contribute to production, as well as to identify the areas with output potential in sandy horizons correlated with the study area.
\end{abstract}

Keywords: log evaluation, petrophysical rock type and flow units. 


\section{Introducción}

La Cuenca Terciaria de Veracruz se localiza al oriente de la República Mexicana y aporta el $35 \%$ de la producción de gas seco en la Región Norte de Pemex Exploración y Producción, dentro de la cuenca se localiza el campo A de donde se extrae el $25 \%$ de la producción de gas seco y se ubica a $85 \mathrm{~km}$ al sureste de la ciudad de Veracruz, Veracruz (Figura 1).

Por la importancia en su producción se realizó la caracterización petrofísica con el objetivo de reevaluar las reservas y buscar acumulación de hidrocarburos con las mismas características de este yacimiento dentro de la cuenca. La producción de hidrocarburos se asocia con sistemas amalgamados de ambientes turbidíticos de edad
Plioceno-Mioceno (Figura 2).

La metodología aplicada consiste en la integración de la geología, geofísica, datos de las pruebas de producción, análisis de registros geofísicos y datos de laboratorio de núcleos como son: porosidad, permeabilidad absoluta, densidad de grano, descripción de láminas delgadas, análisis granulométrico, difracción de rayos $\mathrm{X}$, análisis de microscopio de barrido electrónico, resonancia magnética nuclear y presión capilar con inyección de mercurio (Gunter et al., 1997).

\section{Geología Regional}

El área se ubica en la Cuenca Terciaria de Veracruz, que

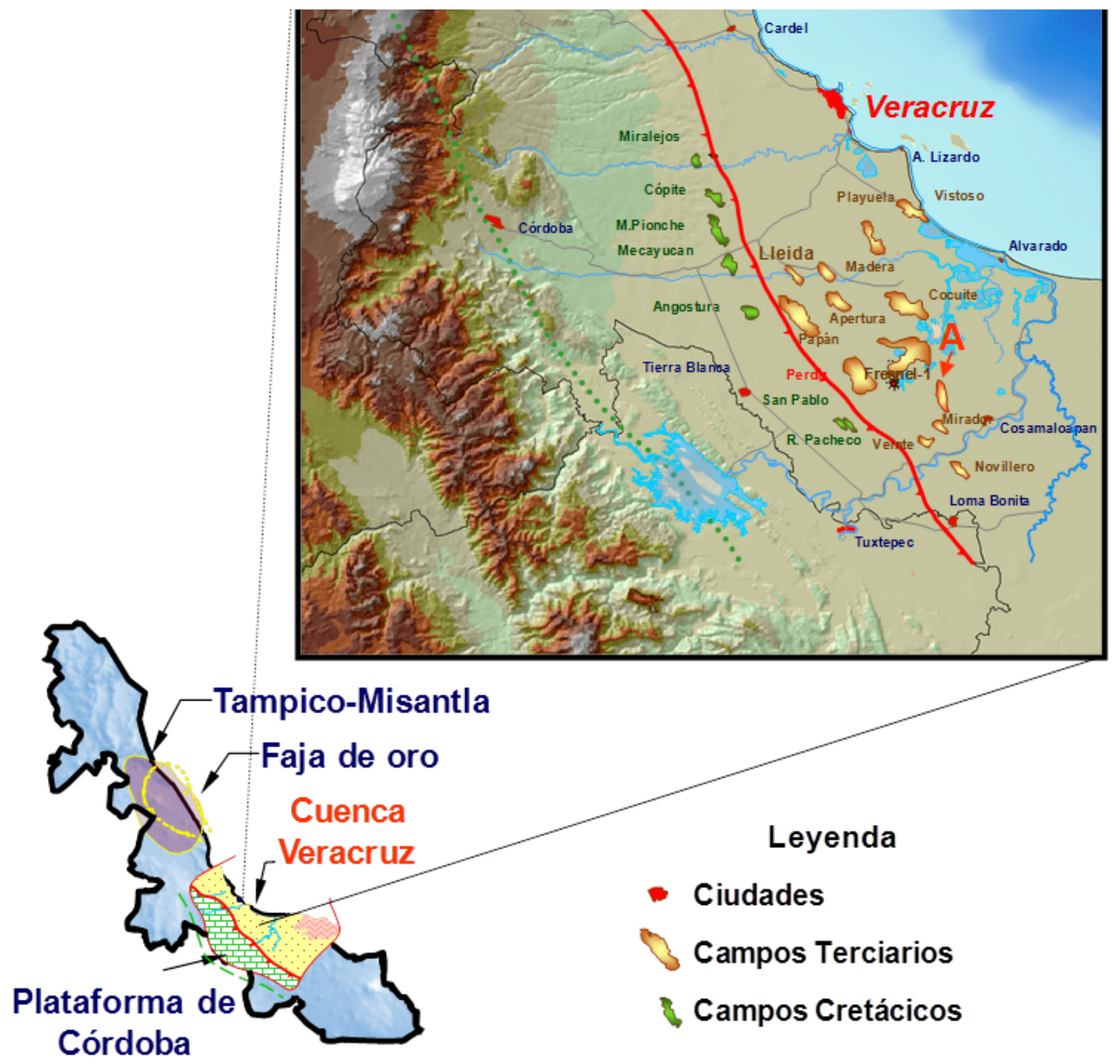

Figura 1. Localización del campo A en la Cuenca Terciaria de Veracruz. 


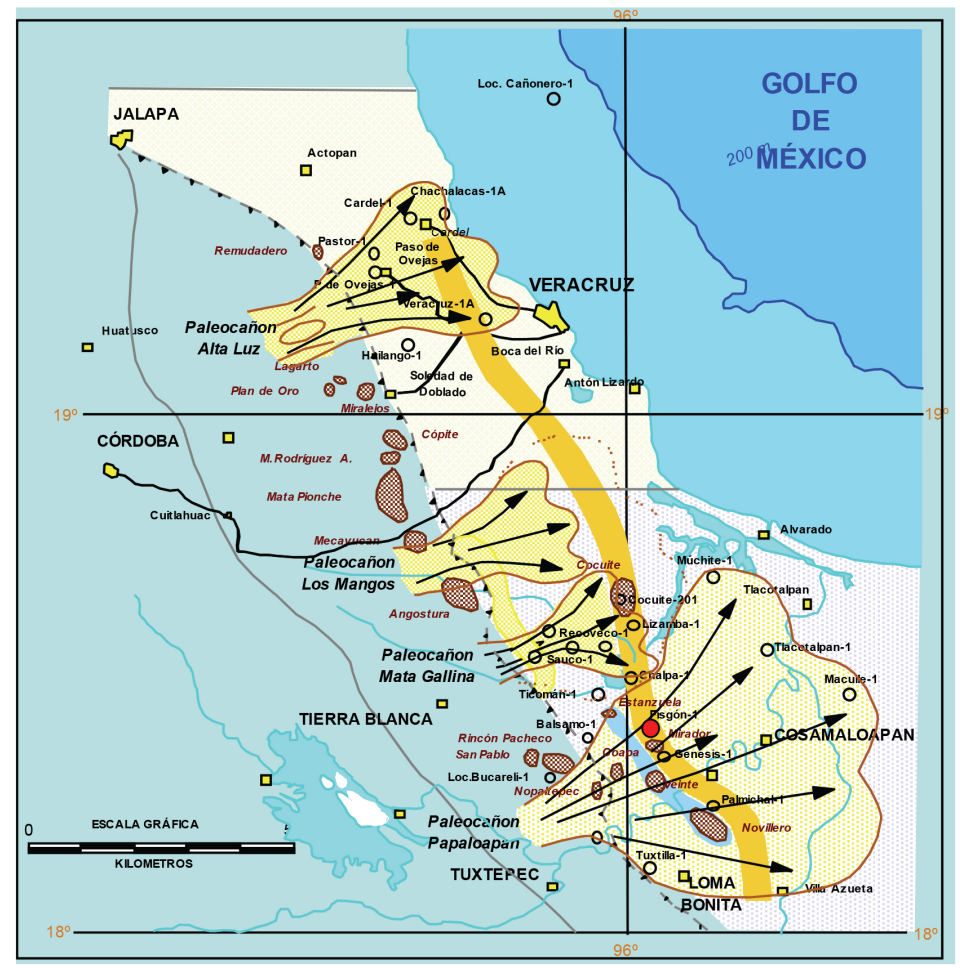

\section{Leyenda}

\section{Poblados}

\section{Campos petroleros}

\section{Alineamiento Novillero-Cocuite-Playuela}

Abanicos Submarinos

Campo A

Figura 2. Abanicos de piso de cuenca en la Cuenca Terciaria de Veracruz durante el Plioceno-Mioceno (Soto-Cuervo, 1999).

corresponde a una gran depresión donde fueron depositados sedimentos terrígenos en forma de abanicos submarinos, deltas, canales, y cuyas edades van del Paleoceno al Reciente (Martínez-Medrano et al., 2006) (Figura 2).

En el Mioceno Medio-Tardío y principios de Plioceno Temprano se depositaron secuencias arcilloso-arenosas, los cuales fueron levantadas, formándose dos alineamientos regionales denominados Novillero-Cocuite-Playuela y el Morillo-Acula-Muchite, la sedimentación en el área continuó y se depositaron abanicos de piso de cuenca los cuales rellenaron las partes bajas y se acuñaron contra los altos previamente formados (Prost y Aranda., 2001, SotoCuervo, 1999).

En los sedimentos que rellenaron la subcuenca entre los dos altos estructurales, se encuentran 5 horizontes de areniscas con diferentes espesores los cuales se acuñan contra el flanco oriental de la estructura Novillero-Cocuite-
Playuela y forman los yacimientos denominados Arquimia MS1, MS2, MS3, MS4 y MS5. La figura 3 es una sección sísmica con rumbo NE-SW mostrando el pozo A-101 en un flanco occidental de un anticlinal, el cual atravesó los 5 cuerpos de arenisca depositados durante el Mioceno Superior y Plioceno Inferior.

A finales del Plioceno Medio y Tardío la sedimentación consistió principalmente de lutitas y funcionan como roca sello para las areniscas del campo A.

\section{Tipos de roca petrofísicos a partir de núcleos convencionales}

En el pozo A-101 se recuperaron 4 núcleos convencionales y 44 núcleos de pared a los cuales se les realizaron análisis para obtener las propiedades petrofísicas 

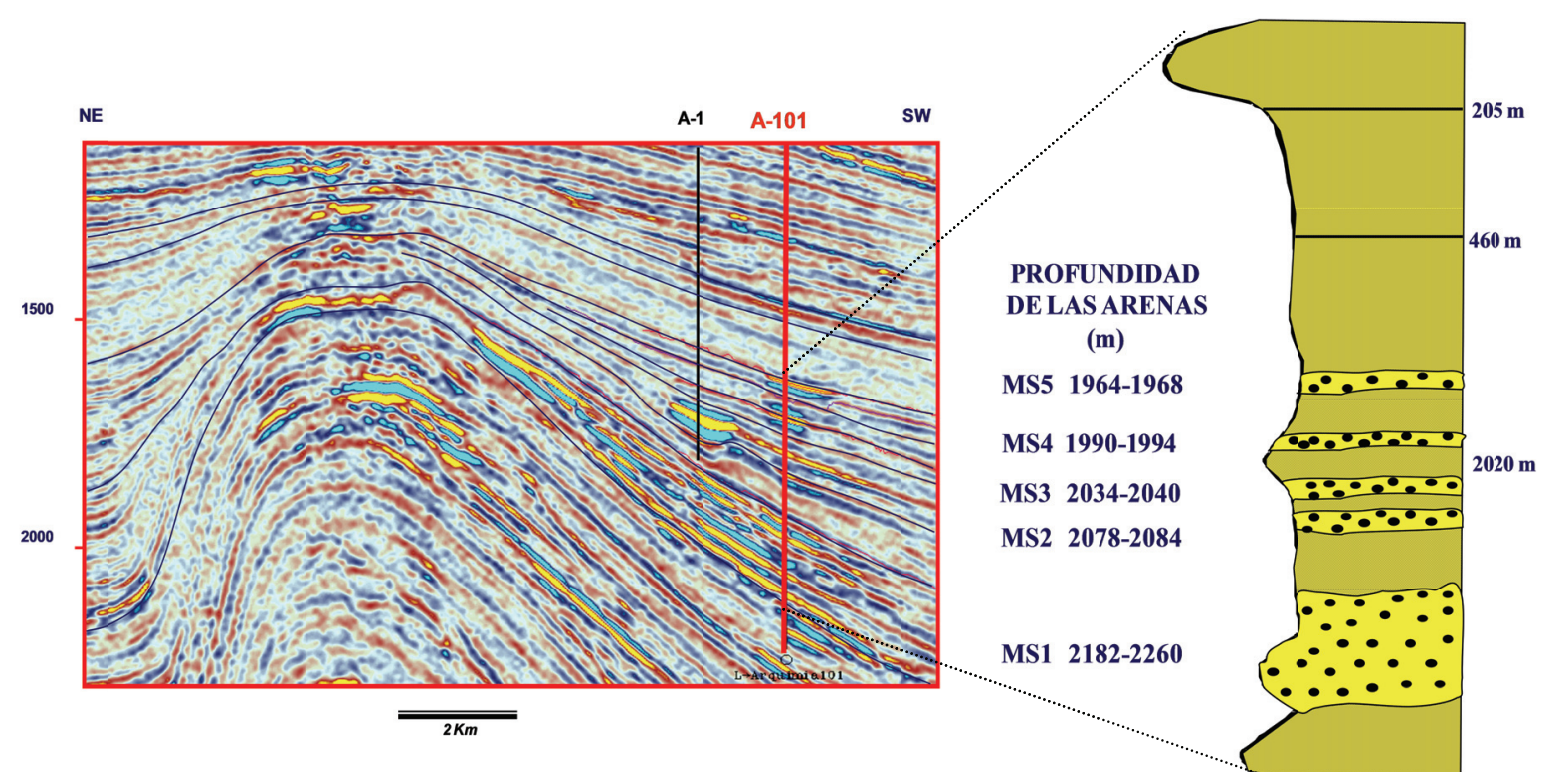

Reciente

Plioceno

Medio

Plioceno

Inferior

Mioceno

Superior

Figura 3. Línea sísmica NE-SW en el campo A, ilustrando el acuñamiento de los depósitos del Neógeno al anticlinal, en el lado derecho la columna geológica atravesada por el pozo A-101 del Reciente al Mioceno Superior

(porosidad, permeabilidad, densidad de grano, tamaño de grano, mineralogía, etc.), con la porosidad y permeabilidad se identifican los tipos de roca petrofísicos. Un tipo de roca petrofísico se definen como: unidades de roca depositadas en condiciones similares que experimentaron procesos diagenéticos similares, dando como resultado una relación única de porosidad y permeabilidad (Gunter et al., 1997). En general, en el análisis de los tipos de roca petrofísicos se puede entender las siguientes características:

1.- El mejor tipo de roca petrofísico presenta baja saturación de agua.

2.- El peor tipo de roca petrofísico la saturación de agua irreducible es alta.

3.- Relación de la geometría de poro y distribución del tamaño determinada con las gráficas de presión capilar.

4.- Se puede determinar la saturación de agua da la altura de agua libre en el yacimiento.

5.-Los tipos de roca petrofísicos se obtienen con el análisis de núcleos y/o evaluación de los registros geofísicos.

Para determinar los tipos de roca se utiliza la ecuación de Winland (Kolodzie, 1980; Martin et al., 1997) donde se calcula el radio de garganta de poro (R35):

$\log (R 35)=0.732+0.588 \log (k)-0.864 \log (\varnothing)$.

Donde:

$R 35$ = radio de garganta de poro correspondiente al $35 \%$ de inyección de mercurio en una gráfica de presión capilar (micrones, $\mu$ ).

$k=$ permeabilidad a condiciones ambientales $(\mathrm{mD})$.

$\varnothing=$ porosidad a condiciones ambientales (\%).

Calculado el $R 35$ se construye un gráfico cruzado de porosidad (\%) vs. permeabilidad (mD) con isolíneas de garganta de poro $(\mu)$ y se establecen divisiones de porosidad entre 4 y $27 \%$ e isolíneas de garganta de poro de $0.1,0.5$,
2.5 y 10 micras, el cual resultan un total de 13 tipos de roca petrofísicos como una medida estándar para cualquier tipo de roca (Gunter et al., 1997).

La figura 4 muestra los 6 tipos de roca (TR2, TR3, TR4, TR7, TR10 y TR13) determinados con datos de núcleos convencionales y de pared cortados en el pozo A-101.

Otro método para obtener el tamaño de poro de la roca es con la prueba de laboratorio denominada inyección de mercurio a altas presiones, comúnmente denominada curva de presión capilar y se genera inyectando mercurio $(\mathrm{Hg})$ a un cilindro de roca con presión, se gráfica el volumen de mercurio inyectado $v s$. la presión con que fue desplazado. Se establece que la presión requerida para inyectar el mercurio es proporcional al tamaño de poros presentes en la muestra analizada. La curva resultante se tiene graficando el volumen acumulativo de mercurio inyectado contra la presión del mercurio (Stout, 1964). Se entiende que el volumen acumulativo de mercurio es igual al volumen de espacio del poro (Stout, 1964; Katz y Thompos, 1987).

La curva de inyección de mercurio es una medida precisa de las interconexiones del tamaño de poro de una roca.

La figura 5 muestra 4 curvas de inyección de mercurio con el tipo de roca determinado con datos de núcleo; se identifica que el tipo 2 y 3 son los mejores tipos de roca petrofísicos pues requieren bajas presiones para inyectar un fluido por su homogeneidad en el tamaño de poro.

Los comportamientos de la curvas de presión capilar y su relación con el tipo de roca (TR) en este estudio se presenta a continuación:

TR2: típica de excelente roca almacén; la presión de entrada es baja indicando poros grandes y la horizontalidad de la curva se interpreta que son de tamaño uniforme (Figura 5). 


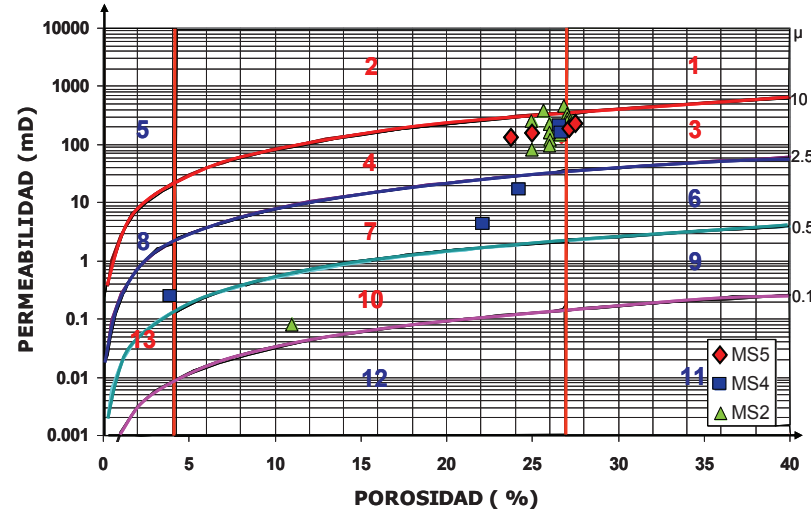

Figura 4. Grafica de Winland, donde se representa los tipos de roca petrofísicos de los núcleos del pozo A-101, los números en rojo representan a los tipos de roca petrofísicos presentes en el pozo

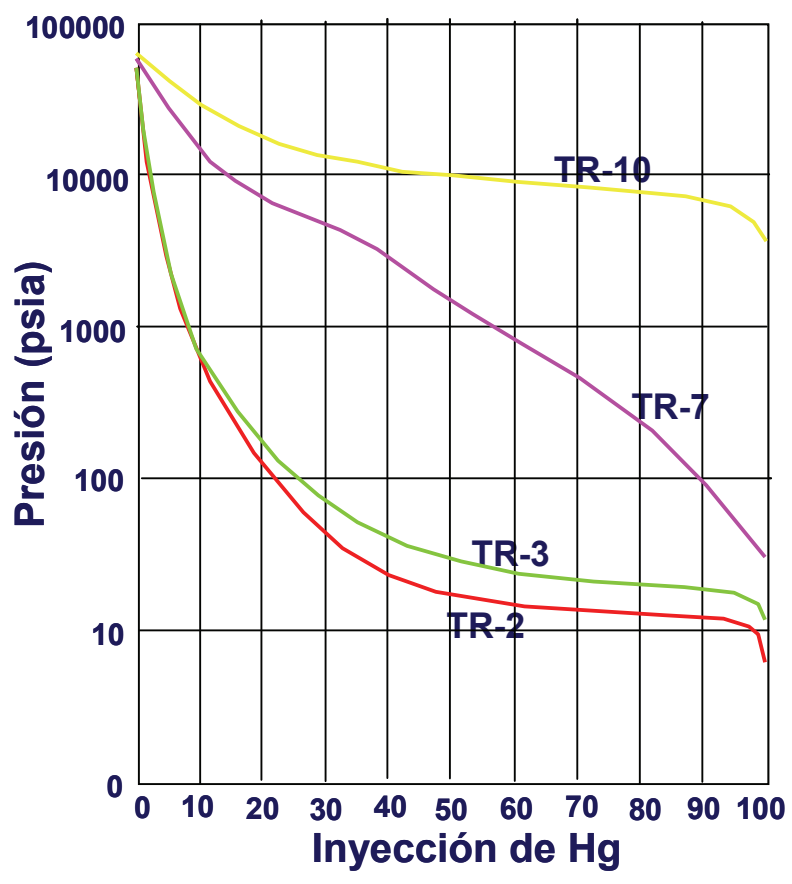

Figura 5. Gráfica de inyección de Hg de los núcleos del pozo A-101.

TR3: el tamaño de poro es menor a la que se observa en la TR2, ya que requiere más presión de entrada; sin embargo sigue siendo un buen tipo de roca (Figura 5).

TR7: es una roca con poros aislados, presenta un aumento constante de la presión con respecto a una inyección mínima de mercurio hasta llegar a un tamaño de poro más grande, y genera una segunda inflexión que es característico de dos tamaños de poros (Figura 5). Este tipo de roca representa el margen mínimo para que una roca almacenadora tuviese producción.

TR10: es típico de roca sello, con una presión alta de entrada e inyección de mercurio muy pequeña, el tamaño de los poros es pequeño (Figura 5).

\section{Tipos de roca petrofísicos a partir de registros geofísicos}

Una vez integrada la información de los núcleos, se realiza una evaluación de los registros geofísicos y se calibra con los datos obtenidos de los núcleos.

A partir de las curvas de porosidad y permeabilidad de los registros editados se realizó la gráfica de Winland, y el resultado fueron los tipos de roca petrofísicos a partir de los registros geofísicos y ahora, se pueden correlacionar en todo el yacimiento.

En la figura 6 están representados los tipos de roca petrofísicos a partir de registros y sus características de porosidad (ø), permeabilidad (k) y radio de garganta de poro (RGP)

En la figura 7 se presentan las láminas delgadas e imágenes del microscopio electrónico de barrido (MEB) correspondientes a los tipos de roca petrofísicos presentes en el yacimiento.

A continuación se describen el tipo de roca petrofísico:

Tipo de roca 1: con un radio de garganta de poro menor a 10 micras, permeabilidad mayor a $300 \mathrm{mD}$ y porosidades de $28 \%$; se clasifica como litarenita de grano fino $(0.17$ $\mathrm{mm}$ ), granos angulares a subredondeados, clasificación moderada, constituida por cuarzo, feldespatos, plagioclasa potásica, fragmentos de calizas y rocas metamórficas; una porosidad intergranular de buen desarrollo, poros amplios; los granos están ligeramente bordeados por arcilla autigénica (illita fibrosa) y siderita. Es el mejor tipo de roca y se corrobora en las gráficas de presión capilar (Figura 6 y 7).

Tipo de roca 3 y 4: tiene un radio de garganta de poro de 2.5 a 10 micras, tiene permeabilidades de 10 a $200 \mathrm{mD}$ y una porosidad promedio de $27 \%$, se clasifica como una litarenita de grano fino a muy fino de granos de cuarzo principalmente, con el microscopio electrónico de barrido (MEB) se observa porosidad de buen desarrollo y arcilla bordeando los granos; la gráfica de inyección de mercurio se muestra como una roca de buena calidad (Figura 6 y 7).

Tipo de roca 7: presenta un radio de garganta de poro de 0.5 a 2.5 micras, con permeabilidades de 5 a $10 \mathrm{mD}$ con una porosidad promedio de un $24 \%$, es una litarenita y la porosidad se ve reducida por el aumento de arcilla en un $50 \%$ con respecto al tipo de roca 3-4. En la gráfica de inyección de mercurio se observa esa heterogeneidad en los poros y en la gráfica de volumen de poros se observa regular la distribución de tamaño de poro, este tipo de roca es de mala calidad, pero suficiente para poder fluir en yacimientos gasíferos (Figura 6 y 7 ).

Tipo de roca 10: tiene un radio de garganta de poro de 0.1 a 0.5 micras, permeabilidades menores a $1 \mathrm{mD}$ y porosidad promedio de $22 \%$, es una limolita arcillosa, y es uno de los peores tipos de roca; la porosidad es obstruida por la arcilla; (Figura 6 y 7 ).

Tipo de roca 12: tiene radio de garganta de poro menores a 0.1 micras, permeabilidades menores a 0.01 
y porosidades entre 15 y $25 \%$, es una típica Lutita y es excelente como roca sello.

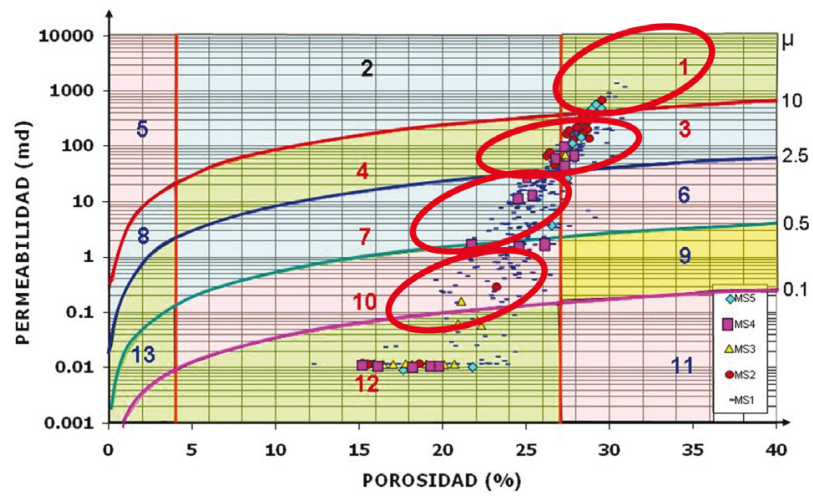

Figura 6. Tipos de roca petrofísicos en los 5 cuerpos de arenisca del Pozo A-101 (Yacimientos MS1, MS2, MS3, MS4 Y MS5).

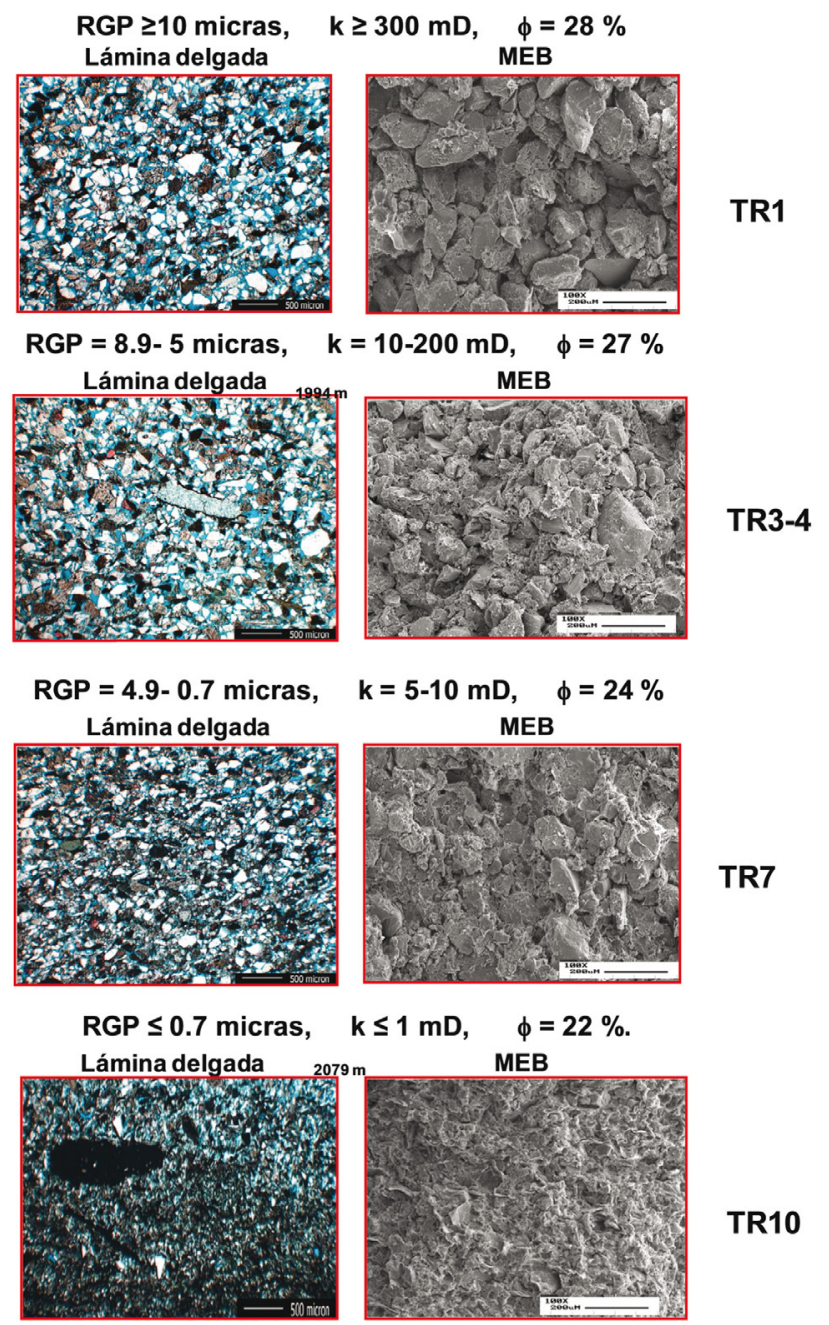

Figura 7. Fotomicrografías (nicoles cruzados) de los diferentes tipos de rocas petrofísicas presentes en el campo, además de una imagen de microscopio electrónico de barrido.

\section{Unidades de flujo}

Una unidad de flujo se define como un intervalo estratigráficamente continuo que respeta el marco geológico y mantiene las características de los tipos de roca (Gunter et al., 1997b).

Para la caracterización petrofísica del yacimiento se subdividió preliminarmente en unidades de flujo considerando los radios de garganta de poro y la frecuencia de porosidad $v s$. permeabilidad.

La gráfica modificada de Lorenz (MLP por sus siglas en ingles) (Craig, 1972) se determina la capacidad de almacenamiento y la capacidad de flujo en porcentaje; para el pozo A-101 se analizó el intervalo 1945-2260 m que comprende los 5 horizontes arenosos previamente mencionados.

La figura 8 es un grafico modificado de Lorenz, en el eje abscisas es la capacidad de almacenamiento (producto de porosidad y espesor, PHIh) y el eje de las ordenadas es la capacidad de flujo (producto de la permeabilidad y espesor, kh) (Chopra et al., 1987); la interpretación del grafico es la siguiente: líneas horizontales son barreras al flujo y las que tienden a ser verticales presentan una buena capacidad de flujo; cabe hacer mención que las barreras al flujo son consideradas como unidades de flujo.

En el intervalo analizado se tienen un total de 13 unidades de flujo de las cuales 7 son barreras al flujo. Las arenas MS1, MS2 y MS5 (franja amarilla) tienen muy buena capacidad de flujo.

La gráfica modificada estratificada de Lorenz (SLMP) (Craig, 1972), muestra en el eje de las abscisas la profundidad y en de las ordenadas la capacidad de flujo, la capacidad de almacenamiento y la curva de almacenamiento de hidrocarburos, la figura 9 se observar que las arenas MS2 y MS5 tienen buena capacidad de flujo y buena capacidad de almacenamiento, mismas que producen el $50 \%$ de la producción de este pozo.

\section{Tipos de fluidos en los yacimientos}

Para predecir los fluidos en el yacimiento fue necesario realizar una evaluación de registros geofísicos, tomando como base los análisis previos de tipos de roca y unidades de flujo. El modelo para el cálculo de la saturación fue el de doble agua, con una salinidad de $30000 \mathrm{ppm}$, la cual fue tomada de las muestras de fluido obtenidas durante la las pruebas de producción del pozo (Figura 10).

En la figura 10 en la parte izquierda (4 carriles) tenemos los registros obtenidos en el pozo y en la parte derecha (4 carriles) los registros interpretados. En los registros tomados del pozo se observa zonas limpias en el registro de rayos gamma (arenas MS1, MS2, MS3, MS4 y MS5) y en esos cuerpos las resistividades son del orden de 2 a $15 \mathrm{ohmm}$, en los registros de litodensidad-neutrón se observan efectos de 


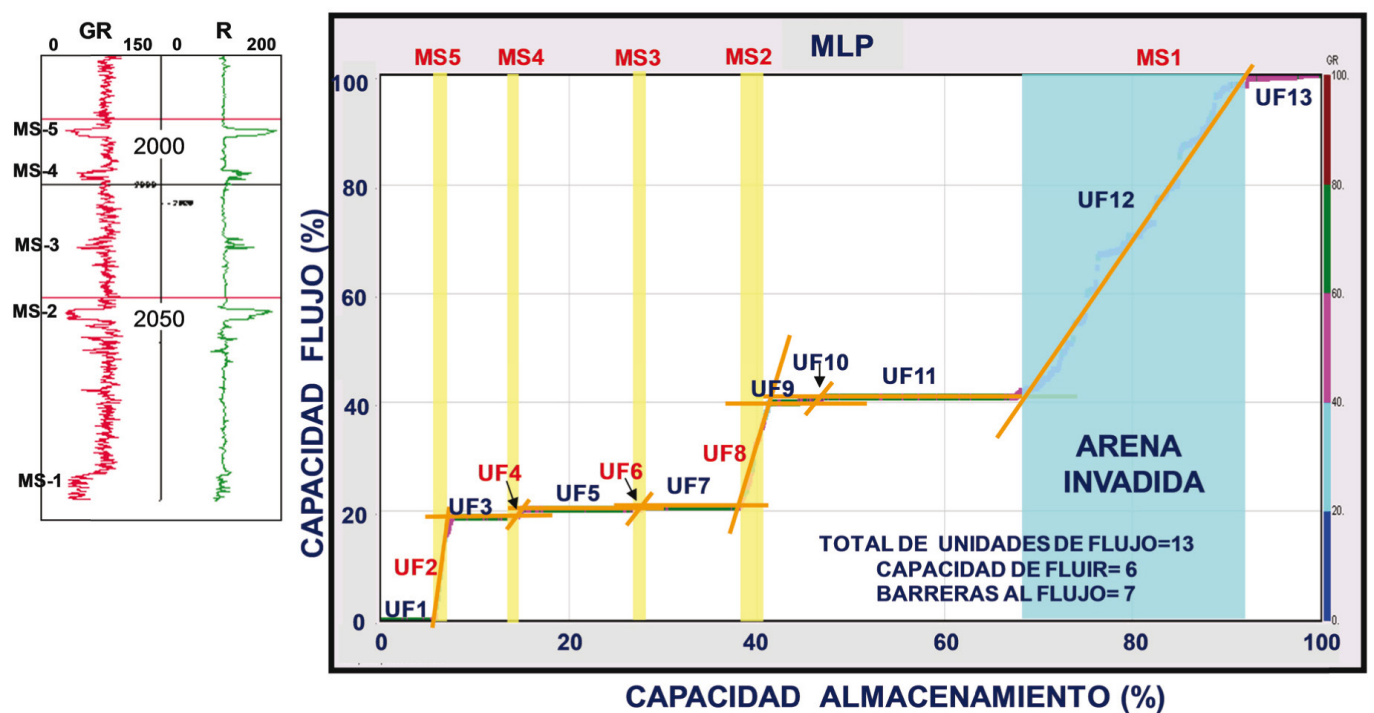

Figura 8. Grafica Modificada de Lorenz (MLP) del pozo A-101, en la parte izquierda se presenta los registros geofísicos rayos gamma, resistividad y los yacimientos (MS1, MS2, MS3, MS4, MS5), en el lado derecho las 13 unidades de flujo en el intervalo 1945 a $2260 \mathrm{~m}$.

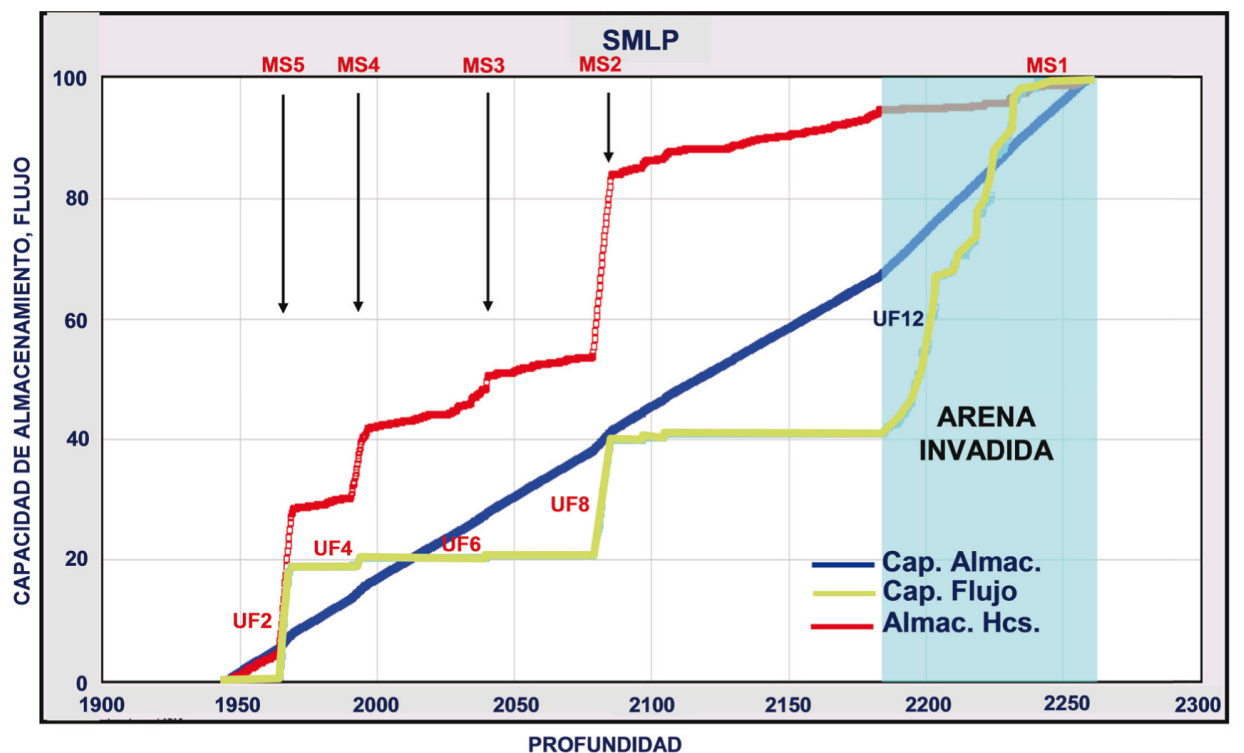

Figura 9. Gráfica Estratificada Modificada de Lorenz (SMLP), en el eje y se presenta la capacidad de almacenamiento y flujo (\%), en el eje x la profundidad (m). La curva roja representa el almacenamiento de hidrocarburos, la curva verde la capacidad de flujo y la curva azul capacidad de almacenamiento, además de las arenas MS1,MS2,MS3,MS4 y MS5.

hidrocarburo ligero en las arenas MS2 y MS5, la tabla 1 es un resumen de las propiedades petrofísicas de cada arena; las arenas MS2 y MS5 tienen las mejores permeabilidades y porosidades, además de saturaciones bajas de agua; para corroborar la interpretación de los registros en los cuerpos de interés; se tomaron puntos de presión y se estableció que en las arenas MS2, MS3, MS4 y MS5 muestran gradientes independientes de gas y la arena MS1 existe un gradiente de agua, concluyendo que son yacimientos independientes.

\section{Características del los yacimientos}

Para determinar las características del yacimiento se utilizó sísmica e ingeniería de yacimientos. Para la sísmica se obtuvo la resolución vertical teniendo la frecuencia dominante y la velocidad de intervalo.

$$
\mathrm{Rv}=\frac{\text { Vint }}{4 * \mathrm{Fd}}
$$

Donde:

Rv: Resolución vertical.

Vint.: Velocidad de intervalo. 


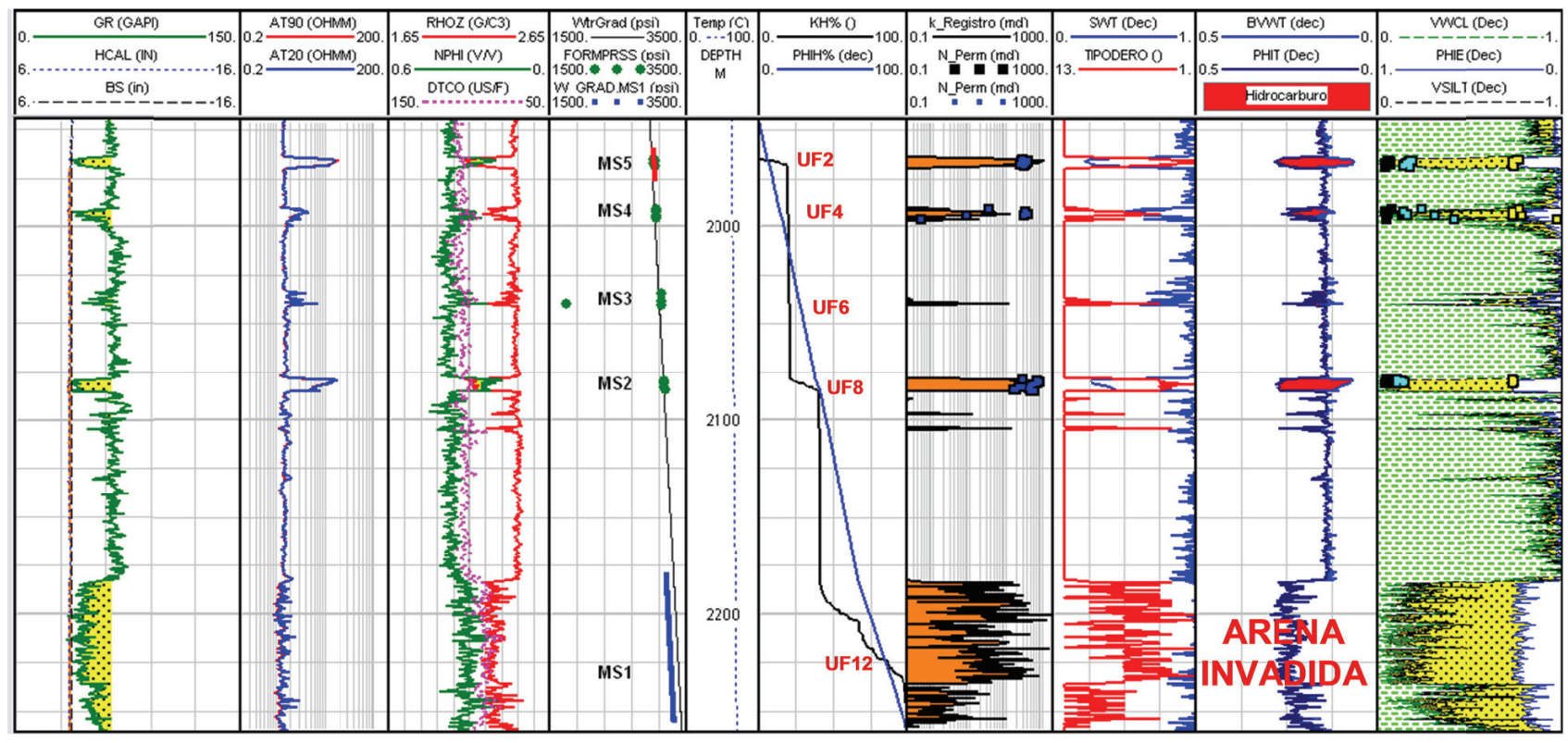

Figura 10. Evaluación de registros del pozo A-101. (Carril 1: rayos gamma, caliper. Carril 2: resistividad (AT90 profunda, AT20 somera). Carril 3: densidad (RHOZ), porosidad (NPHI), sónico compresional (DTCO). Carril 4: presiones. Carril 5: profundidad (Depth en m), Temperatura (Temp). Carril 6: unidades de flujo (Kh), capacidad de almacenamiento (PHIh). Carril 7: permeabilidad de registros (k_registros, perm. de núcleos (N_perm). Carril 8: saturación de agua (SW), tipos de roca. Carril 9: Vol. de agua total (BVWT), porosidad total (PHIT). Carril 10: litología).

Tabla 1. Características de los diferentes tipos de roca (RT: tipo de roca, k: permeabilidad, Phi: porosidad, h: altura neto impregnado).

\begin{tabular}{ccccc}
\hline RT & RT (\%) & k (md) & Phi (\%) & h (ft) \\
\hline 1 & 37.5 & 374.5 & 28.01 & 4.92 \\
3 & 25 & 261.2 & 27.46 & 3.28 \\
4 & 18.75 & 111.9 & 26.36 & 2.46 \\
7 & 12.5 & 15.37 & 25.9 & 1.64 \\
10 & 6.25 & 0.0036 & 20.7 & 0.82 \\
\hline
\end{tabular}

Fd: Frecuencia dominante.

La resolución vertical de la símica es de $28.2 \mathrm{~m}$, con esta resolución no se alcanza a observar cuerpos de arenisca de este pozo ya que en promedio tienen solo 4-6 m de espesor, pero en este caso especifico se alcanza observar por que las areniscas tienen presencia de gas y su contraste de densidades es muy fuerte (O'Brien, 2004).

En la figura 11 se presenta una línea sísmica donde se ubican los pozos A-1 y A-101, se observan las fuertes reflexiones el cual se interpreta como cuerpos arenosos y son observadas por el contraste de densidades de roca-fluido.

Con el objetivo de calibrar la sísmica con los registros geofísicos se realizaron 4 gráficas que se describen a continuación y están representados en la figura 12:

La primera gráfica es la litodensidad-neutrón (densidad $v s$. porosidad) y nos muestra un efecto de hidrocarburo ligero (gas), además como un tercer componente se observa donde se encuentran las lutitas y las probables areniscas cargadas con gas (Figura 12). La segunda gráfica es impedancia acústica compresional (Zco), vs. impedancia acústica de cizalla (Zsh). La impedancia acústica es el producto de la

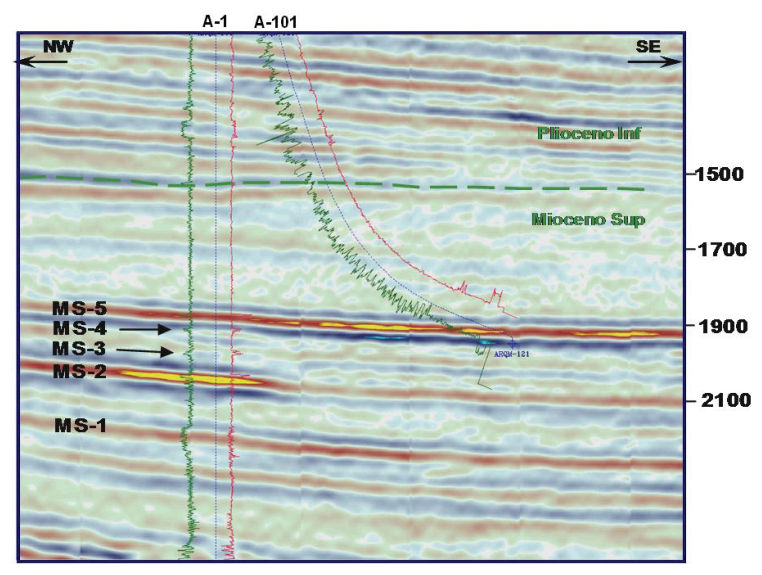

Figura 11. Sección sísmica NW-SE en el campo A y los cuerpos de areniscas cortados.

densidad y la velocidad, esto es, a mayor densidad mayor velocidad y viceversa. La impedancia acústica es un atributo de la sísmica que nos da cualitativamente zonas porosas de interés y funciona bajo el principio de una mayor impedancia acústica menor porosidad y a menor impedancia acústica mayor porosidad. Por tal motivo podemos inferir donde están las areniscas, que probablemente estén cargadas con hidrocarburo. Los valores de impedancia acústica compresional en las arenas con gas son de 19000 y 23000 , para la impedancia acústica de cizalla entre 11000 y 13000 (Figura 12).

En la tercer gráfica de impedancia acústica compresional (Zco) vs. relación de Poisson (PR). La relación de Poisson se define como un cambio lateral de un cuerpo con respecto 

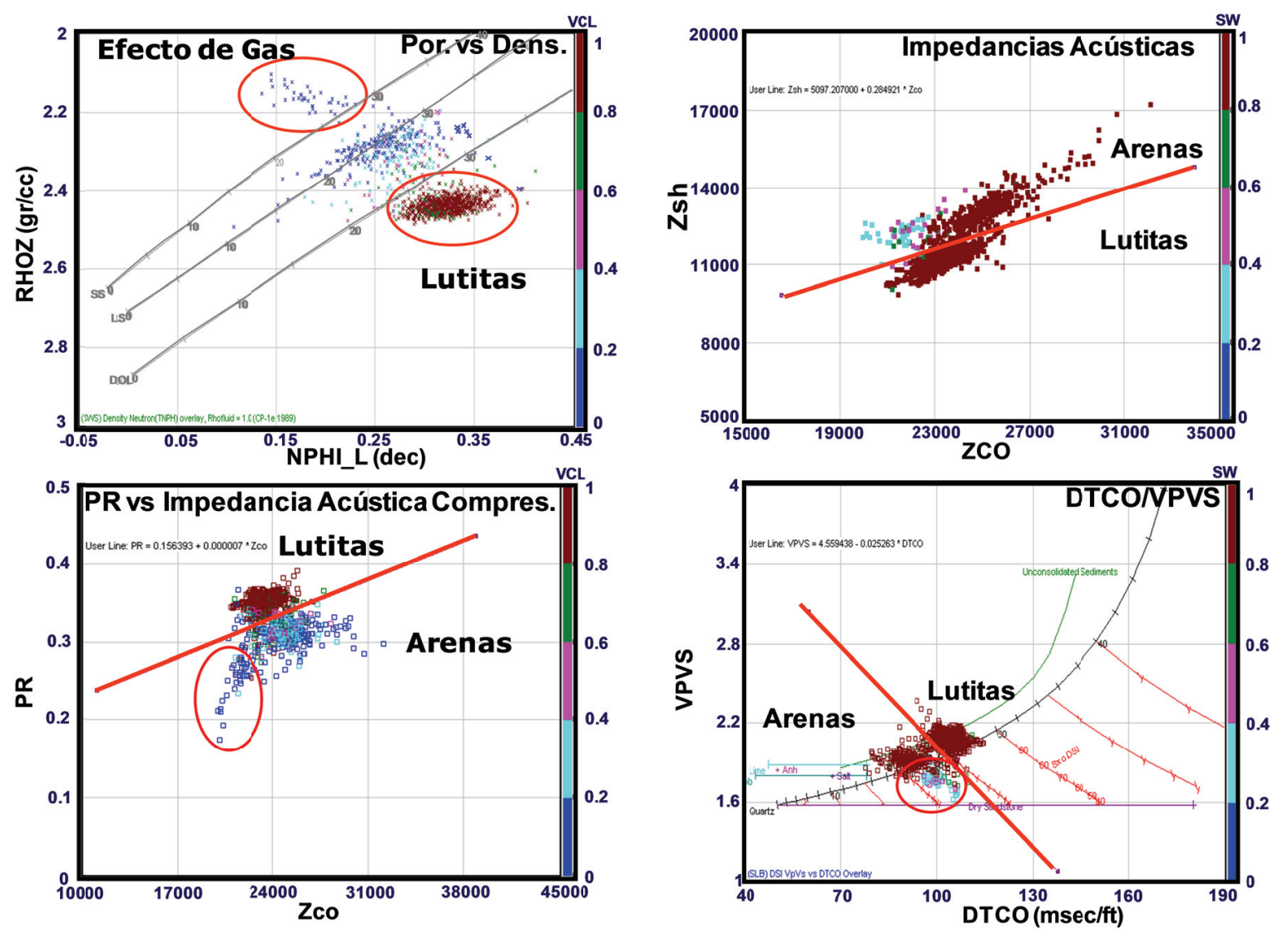

Figura 12. Gráficas porosidad vs. densidad (superior-izquierda), impedancia acústica compresional vs. cizalla (superior-derecha), impedancia acústica vs. relación de poisson (inferior-izquierda), sónico compresional $v s$. relación VPVS (inferior-derecha).

a su respectivo cambio longitudinal, bajó la acción de un esfuerzo. Se tienen valores característicos para diferentes litología, entre más bajo es la relación de Poisson más compacta es la roca, para este estudio se tienen valores de relación de Poisson para las areniscas de 0.30 y para las lutitas 0.35 y como tercer componente se tiene el volumen de arcilla ( $\mathrm{Vcl}$ ) donde podemos definir los cuerpos arenosos y los cuerpos arcillosos (Figura 12).

En la cuarta gráfica se presentan los valores del registro sónico compresional (DTCO) vs. la relación VPVS, además de observar las arenas cargadas con gas también nos muestra el grado de compactación de la roca (Figura 12).

Estas gráficas son muy importantes porque podemos mapear y calibrar las propiedades petrofísicas y geomecánicas de la roca como porosidad, densidad de roca, relación de Poisson, impedancia acústica, relación $\mathrm{Vp} / \mathrm{Vs}$ en un cubo sísmico y buscar rocas con el mismo comportamiento para la localización de hidrocarburos.

\section{Yacimientos}

El yacimiento es de gas seco, el mecanismo de empuje roca-fluido, en las pruebas de producción las presiones oscilan entre 2150 a 2700 psi.

Se realizó un cálculo del gasto y posteriormente el factor de recuperación (FR) para cada tipo de roca, por arena o unidad de flujo con la siguiente ecuación.

$$
\mathrm{Fr}=1-\frac{\mathrm{Bgi}}{\mathrm{Bga}}
$$

Donde:

Fr=Factor de recuperación

$\mathrm{Bgi}=$ Volumen de gas inicial

$\mathrm{Bga}=$ Volumen de gas de abandono.

El gasto calculado es muy cercano del que se tiene en la prueba de producción ya medida en campo. El factor de recuperación se estimó teniendo el volumen de gas inicial y de abandono que fue de $67.5 \%$.

\section{Discusión}

En el campo A existen 7 pozos de los cuales se analizó solamente uno, porque presenta la mejor información del campo. Es difícil realizar la caracterización petrofísica con solo pozo, pero el comportamiento de los cuerpos arenosos es muy similar en este caso. 
Una de las principales incertidumbres en los modelos petrofísicos es extrapolar los parámetros petrofísicos (porosidad, permeabilidad y saturación de agua) a diferentes cuerpos arenosos. En un principio se busco analizar la roca por su clasificación o sus facies, pero genera mayor incertidumbre ya que la diagénesis juega un papel muy importante en las características de cada roca. Por tal motivo se determinaron los tipos de roca petrofísicos y las unidades de flujo ya que pueden extrapolarse en todo el yacimiento.

Otro punto importante es ¿Cómo deben ser tratadas las arenas del campo A, como diferentes yacimientos o uno solo?, la respuesta se encontró en los datos de puntos de presión que fueron tomados durante la perforación, ya que cada cuerpo arenoso muestra un gradiente de gas diferente, $o$ sea no están conectados, por tal motivo los cuerpos arenosos deben ser tratados como yacimientos independientes.

\section{Conclusiones}

Se realizó la caracterización petrofísica del campo A en la Cuenca Terciaria de Veracruz en areniscas del Mioceno Superior y Plioceno Inferior, el cual ayudó a reevaluar las reservas del campo, optimizar la explotación de los yacimientos, obtener un modelo estático más confiable y sirvió de base a modelo de simulación (modelo dinámico).

La caracterización petrofísica permitió implementar ajustes en las estrategias de desarrollo como la perforación de pozos horizontales, obteniendo 3 veces más de producción con lo que respecta a un pozo vertical. También permitió buscar nuevas acumulaciones de hidrocarburos con las mismas características petrofísicas y geomecánicas en área más al norte de la estructura localizando pequeños cuerpos cargados con hidrocarburos.

En las areniscas MS2 y MS5 se observa presencia de gas en las gráficas, Densidad-Neutrón y DTCO y VPVS.

Se determinaron 7 tipos de roca de las cuales las RT1, RT3, RT4, RT7, son las que mejor contribuyen a la producción de gas.

Se tienen 13 unidades de flujo en todo el campo y se determinaron 4 como productoras principales.

\section{Recomendaciones}

Se recomienda realizar una base de datos de las características de impedancias acústicas, relación de Poisson, relaciones VPVS, etc. para realizar análisis probabilísticos

Los cuerpos de areniscas del campo A son yacimientos independientes por lo que se recomienda que sean explotados como tal.

\section{Agradecimientos}

Un agradecimiento a PEMEX Exploración y Producción por permitir transmitir esta información en especial al Ing. Antonio Escalera Alcocer; a los Ings. Arturo Soto Cuervo, Antonio Cuevas Leree, Antonio Hernández Patiño, Ramón Cárdenas Hernández. Un agradecimiento a Bill Brayan, Gary Gunter, Jhon Doveton y Eduardo Viro por su valiosa contribución a este trabajo. Al Dr. Claudio Bartolini por sus extraordinarias correcciones, al Ing. Luis Rey Rosas Flores por la revisión técnica y críticas para el mejoramiento del mismo.

\section{Referencias}

Chopra, A.K., Stein, M,H., Ader, J.C., 1987, Development of Reservoir Descriptions to Aid in Design of EOR Projects: Society of Petroleum Engineers, 4 (2), 143-150.

Craig, F.F., 1972, The Reservoir Engineering Aspects of Waterflooding: Society of Petroleum Engineers, SPE Monograph 3, 63-66.

Gunter, G., Pinch, J.J., Finneran, J.M., Bryant, W.T., 1997a, Overview of an Integrated Process Model to Develop Petrophysical Based Reservoir Descriptions: Society of Petroleum Engineers 38748, 475-479.

Gunter, G., Finneran, Hartman, D,J., Miller, J, D., 1997b, Early determination of reservoir flow units using an integrated Petrophysical Method, Society of Petroleum Engineers 38679, 373-380.

Katz, A.J., Thompson, A.H., 1987, Prediction of rock electrical conductivity from mercury injection measurements: Journal of Geophysical Research, 92, 599-607.

Kolodzie, S.J., 1980, The Analysis of pore Throat Size and Use of WaxmanSmit to Determine OOIP in Spindle Field, Colorado: Society of Petroleum Engineers 9382.

Martin, A.J., Salomon, S.T., Hartmann, D.J., 1997, Characterization of Petrophysical Flow Units in Carbonate Reservoirs: American Association of Petroleum Geologists Bulletin, 81, 734-759.

Martínez-Medrano, M., Vázquez-Benítez, R., Valdivieso-Ramos, V.M., Arreguin-López, M.A. Rivera-Cruz, S., 2006, Upper MiocenePliocene Plays and their Economical Important in the Tertiary Veracruz Basin, Mexico: The Gulf Coast Association of Geological Societies, 56, 575.

O'Brien, J., 2004, Seismic Amplitudes from low gas saturation sands, The Leading Edge, 23, 1236-1243.

Prost, G., Aranda, M., 2001, Tectonics and hydrocarbon systems of the Veracruz, Basin, México, en Bartolini, C., Buffler, R.T., CantúChapa, A. (eds.), The Western Gulf of Mexico Basin: Tectonics, sedimentary basins, and petroleum systems: American Association of Petroleum Geologists, Memoir 75, 271-291.

Soto-Cuervo, A., 1999, Oportunidades exploratorias Localizaciones a perforar del Terciario. Residencia de Exploración Veracruz (Inédita).

Stout, J.L., 1964, Pore geometry as related to carbonate stratigraphic traps,American Association of Petroleum Geologists Bulletin, 48, 329-337.

Manuscrito recibido: Mayo 16, 2010.

Manuscrito corregido recibido: Marzo 27, 2011.

Manuscrito aceptado: Abril 22, 2011. 